\title{
Photothermal Investigation of Surface Defects of Pure Semiconducting A2B6 Materials
}

\author{
J. Zakrzewski - M. Maliński - K. Strzałkowski • \\ D. Madaj · F. Firszt • S. Lęgowski · H. Męczyńska
}

Received: 28 September 2011 / Accepted: 12 April 2012 / Published online: 25 April 2012

(C) The Author(s) 2012. This article is published with open access at Springerlink.com

\begin{abstract}
Photoacoustic spectroscopy is a sensitive and useful method to investigate the quality of semiconducting A2B6 crystals. An imperfection of surface quality can strongly influence photoacoustic spectra but it shows the different character for the different kinds of semiconducting materials. To properly interpret the amplitude and phase spectra, the temperature distribution and its modifications, due to the surface defects, are needed. The Blonskij model of the temperature distribution was used to investigate the influence of the defect on the amplitude and phase spectra.
\end{abstract}

Keywords A2B6 materials · Photoacoustic spectroscopy - Piezoelectric detection · Semiconductors

\section{Introduction}

Photothermal methods have become a very useful tool for measurements of the optical and thermal parameters of semiconductors [1-4]. They have also been used to investigate the surface properties of different materials [5-8]. The significance of thermal phenomena becomes more and more important because of the problem of energy

\footnotetext{
J. Zakrzewski $(\varangle) \cdot$ K. Strzałkowski · F. Firszt · S. Łęgowski · H. Męczyńska Instytut Fizyki, Uniwersytet Mikołaja Kopernika, ul. Grudziądzka 5/7, 87-100 Torun, Poland e-mail: jzakrzew@fizyka.umk.pl

\section{Maliński}

Department of Electronics, Technical University of Koszalin, ul. Sniadeckich 2, 75-328 Koszalin, Poland

D. Madaj

Wydział Matematyki i Informatyki, Uniwersytet Warmińsko-Mazurski, ul. Żołnierska 14, 10-561 Olsztyn, Poland
} 
dissipation in miniaturized semiconducting devices. One of the thermal methods is the piezoelectric (PZE) photothermal technique which was theoretically described by Jackson and Amer (J-A) [9] and by Blonskij et al. [10]. The J-A theory was successfully used to investigate the properties of A2B6 mixed crystals [11-13].

Wide-bandgap A2B6 compounds are still the subject of intense research, mainly because of their application in bandgap engineering, interband and intersubband transitions, and dilute semiconductors, materials, and devices. Among others, ZnSe appear promising as a wide-bandgap semiconductor for applications in high power/high-frequency devices, blue and ultraviolet light-emitting devices/photodetectors, and chemically stable substrates for epitaxial growth of various materials. Cadmium telluride $(\mathrm{CdTe})$ is one of the promising materials for high efficiency, low-cost thin-film solar cells. CdS is useful for many significant applications such as light-emitting diodes, flat panel displays, solar cells, photocatalysts, and thin-film transistors. The mechanical and optical properties of single crystals depend on various factors such as the quality of the starting material, the heat treatment process, the pulling rate of the ampoule, and the growth temperature.

For all of these materials it is possible to change electronic properties, lattice parameters, and bandgap energies by adjusting the composition of the mixed crystals. The change of the bandgap energy gives the desired optical properties and emission over the entire visible and ultraviolet ranges of the spectrum.

Recently, very interesting features have been observed, namely, for many kinds of mixed crystals, the preparation process strongly influencing the photoacoustic spectra [13]. The aim of this article is to present and interpret the influence of surface defects on the PZE amplitude and phase spectra of some groups of A2B6 pure compounds.

\section{Sample Preparation}

All the samples were grown from high purity powder with the high-pressure Bridgman method. For all materials, the crystal rods were cut into about $1 \mathrm{~mm}$ thick samples which were first ground using grinding powder (diameter of $10 \mu \mathrm{m}$ ). The samples were then polished with diamond paste $(1 \mu \mathrm{m})$ and finally chemically etched. A solution of $\mathrm{H}_{2} \mathrm{SO}_{4}(96 \%), \mathrm{K}_{2} \mathrm{Cr}_{2} \mathrm{O}_{7}$, and water was used for etching the samples. After etching, the samples were rinsed in distilled water and then put in boiling $\mathrm{NaOH}$ for a few seconds. Then the samples were rinsed again in cold water, next in boiling distilled water, and finally in ethyl alcohol.

\section{Photoacoustic Spectra-Theoretical Predictions}

The basic theory of piezoelectric detection was developed by Jackson and Amer (J-A) [9] and Blonskij et al. [10]. The amplitude and phase piezoelectric spectra can be computed for the rear experimental configuration with the expression given by the one-dimensional (1D) formula [14], which is a modified J-A expression: 


$$
S \cong \frac{1}{l} \int_{-l / 2}^{l / 2} T(x) \mathrm{d} x-\frac{6}{l^{2}} \int_{-l / 2}^{l / 2} x T(x) \mathrm{d} x
$$

where $l$ is the thickness of a sample, $T(x)$ is the instantaneous temperature spatial distribution, and $x$ is the spatial coordinate.

The temperature distribution in the sample is the basis for the finding of the theoretical amplitude and phase photoacoustic spectra. It is given by the J-A theory and that modified by Maliński et al. [11]. This method was successfully used for the interpretation of PA spectra for A2B6 mixed crystals [11-13]. It gives very good agreement with the experimental data if one assumes the presence of defects in the sub-bandgap region but is not so accurate in the region very close to the value of the energy gap of a semiconducting material.

In the present paper, the temperature distribution developed by Blonskij et al. [10] was chosen to simulate the theoretical spectra and was compared to experimental data. In this simplified model the temperature in the $Y Z$ plane (surface of the sample) is averaged and an expression of the temperature at any point of the sample's thickness is given by

$$
\begin{aligned}
T(x)= & \frac{I \pi b^{2} \beta}{k \sigma\left(\beta^{2}-\sigma^{2}\right)} \\
& \times \frac{\beta \cosh (\sigma(l / 2+x))-\beta \mathrm{e}^{-\beta l} \cosh (\sigma(1 / 2-x))-\sigma \mathrm{e}^{-\beta(l / 2-x)} \sinh (\sigma l)}{\sinh (\sigma l)}
\end{aligned}
$$

where $\alpha$ is the thermal diffusivity of the sample, $\sigma^{2}=\frac{\mathrm{i} \omega}{\alpha}, k$ is the thermal conductivity, $l$ is the sample thickness, $\beta$ is the absorption coefficient, $I$ and $b$ are fixed initial values, $\omega=2 \pi f$, and $f$ is the frequency of modulation.

The next assumption to be carried out is the dependence of the absorption coefficient. Typical ones were taken into account [8]:

$$
\begin{array}{ll}
\beta=\beta_{0} \exp \left(\frac{\left(E-E_{\mathrm{g}}\right) \gamma}{0.025}\right) & \text { for } E \leq E_{\mathrm{g}} \\
\beta=A_{0}\left(E-E_{\mathrm{g}}\right)^{1 / 2} & \text { for } E>E_{\mathrm{g}}
\end{array}
$$

The first equation describes Urbach tail thermal broadening (this is represented by parameter $\gamma$ ) of the absorption band observed for all direct electron type transition semiconductors; the second one represents the absorption band connected with direct, band-to-band, electron transitions in semiconductors.

The above assumptions are sufficient for ideal crystals and surfaces, but modifications must be taken into account for the case of imperfect materials. As was shown for mixed crystals [13-15], one must consider periodic temperature fields generated in the sample caused by the absorption in the volume and at the surfaces of the samples. One must assume the presence of a very thin layer of thickness $d$ with a thermal conductivity different from the volume of the sample. Then two types of thermal distribution 
must be taken into account. One of them is associated with the volume absorption, $T_{V}$, and the other is connected with the surface absorption at one of the surfaces of the sample, $T_{\mathrm{SL}}$ or $T_{\mathrm{SR}}$. Defects located at different surfaces exert different types of influence on the photoacoustic signal. The temperature distribution in the sample can be expressed by the following equations depending on the location of the surface defects for Blonskij's model:

$$
\begin{aligned}
& T_{\mathrm{L}}(x, \beta)=T_{\mathrm{SL}}(x, \beta)+T_{V}(x, \beta) \\
& T_{\mathrm{R}}(x, \beta)=T_{\mathrm{SR}}(x, \beta)+T_{V}(x, \beta)
\end{aligned}
$$

where $T_{\mathrm{SL}}$ is the temperature distribution associated with the surface defects located on the illuminated side of the sample, $T_{\mathrm{SR}}$ is the analogous distribution but associated with the defects located on the opposite side of the sample, and $T_{V}$ is the temperature distribution associated with the volume absorption of light in the sample. The temperature distribution for the surface, taking into account the fact that in that region the absorption takes place only at the surface layer of the thickness $d$ (the volume of the sample is transparent for radiation), is as follows:

$$
\begin{aligned}
& T_{\mathrm{SL}}(x)=\frac{I_{0}(1-\exp (-\beta d))}{k_{\mathrm{S}} \sigma_{\mathrm{S}}} \frac{\left[\cosh \left(\sigma_{\mathrm{s}}(1 / 2+x)\right)\right]}{\sinh \left(\sigma_{\mathrm{s}} l\right)} \\
& T_{\mathrm{SR}}(x)=\frac{I_{0}(1-\exp (-\beta d))}{k_{\mathrm{S}} \sigma_{\mathrm{S}}} \frac{\left[\cosh \left(\sigma_{\mathrm{S}}(1 / 2-x)\right)\right]}{\sinh \left(\sigma_{\mathrm{s}} l\right)}
\end{aligned}
$$

where $k_{\mathrm{S}}$ and $\sigma_{\mathrm{s}}$ characterize thermal parameters of the layer.

The absorption coefficient due to the presence of the defects has a Gaussian character:

$$
\beta=A \exp \left(\frac{E-E_{\mathrm{d}}}{\beta_{1}}\right)^{2}
$$

where $E_{\mathrm{d}}$ is the value of the energy of the defect, $\beta_{1}$ is the parameter describing the width of the Gaussian shape maximum, and $A$ is the amplitude of the maximum.

One must add the modification of the piezoelectric signal $S_{\mathrm{SR} / \mathrm{SL}}$ originated from the defect's presence on illuminated (SR) or non-illuminated (SL) surfaces:

$$
S_{\mathrm{SR} / \mathrm{SL}} \cong \frac{1}{l} \int_{-l / 2}^{l / 2} T_{\mathrm{SR} / \mathrm{SL}}(x) \mathrm{d} x-\frac{6}{l^{2}} \int_{-l / 2}^{l / 2} x T_{\mathrm{SR} / \mathrm{SL}}(x) \mathrm{d} x
$$

In the interpretation of piezoelectric spectra, one must consider the effects due to the above modifications as the sum of $S$ and $S_{\mathrm{SR} / \mathrm{SL}}$. 


\section{Experimental Results and Theoretical Simulations}

Figure 1a, b presents the theoretical predictions (solid line) and experimental data for the amplitude and phase spectra in the rear mode [14] configuration for a CdS sample. For the rear mode method the sample is irradiated from one side and the signal is collected from the other. The values of the parameters are $E_{\mathrm{g}}=2.48 \mathrm{eV}$, thermal diffusivity $\alpha=0.1 \mathrm{~cm}^{2} \cdot \mathrm{s}^{-1}, f=126 \mathrm{~Hz}, \gamma=0.6$, and $l=1 \mathrm{~mm}$. A low value of $\gamma$ which characterizes the thermal broadening of the Urban tail provides not good quality of the crystal. A characteristic peak in the sub-bandgap region in the amplitude spectrum is clearly visible in a simulation (solid line). According to the J-A theory, the peak is due to subtracting the components coming from the piston and drum effects in the rear configuration mode. In the phase spectra this phenomenon is manifested as a change in the phase (value equal to zero) when the compensation for bending and expansion of the sample due to heating occurs. In the experimental data (dashed line) one observes the higher value of the peak in the sub-bandgap region, the characteristic deflection due to the energy gap value is moved to the higher energy. Figure $2 a, b$ shows the corrected simulation in the presence of a surface defect of $E_{\mathrm{d}}=2.32 \mathrm{eV}$ (at illuminated surface) and the layer of the thickness $d=0.1 \mathrm{~mm}$. In this case the temperature distribution for $T_{\mathrm{L}}(x, \beta)$ given by Eq. 5 was used. The intensity of the maximum in the amplitude spectra depends on the value of the Gaussian maximum associated with the defect, as well as the thermal diffusivity of the surface layer, which decreases. Both the amplitude and phase spectra show good agreement with the experiment. It should be noted that the thickness of the layer taken into account is approximate; corrected values can be estimated after comparison to other methods.

The amplitude in the high absorption region (above $E_{\mathrm{g}}$ ) increases. This effect can be connected with the plasma wave [15].

Figure $3 \mathrm{a}, \mathrm{b}$ presents simulation (solid line) and experimental data (dashed line) for the sample of CdSe. The values of the parameters are: $E_{\mathrm{g}}=1.71 \mathrm{eV}$ (literature value of energy gap of CdSe), thermal diffusivity $\alpha=0.04 \mathrm{~cm}^{2} \cdot \mathrm{s}^{-1}, f=126 \mathrm{~Hz}$, $\gamma=0.6$ (presumed not good quality of the crystal), and $l=1 \mathrm{~mm}$. Both the amplitude
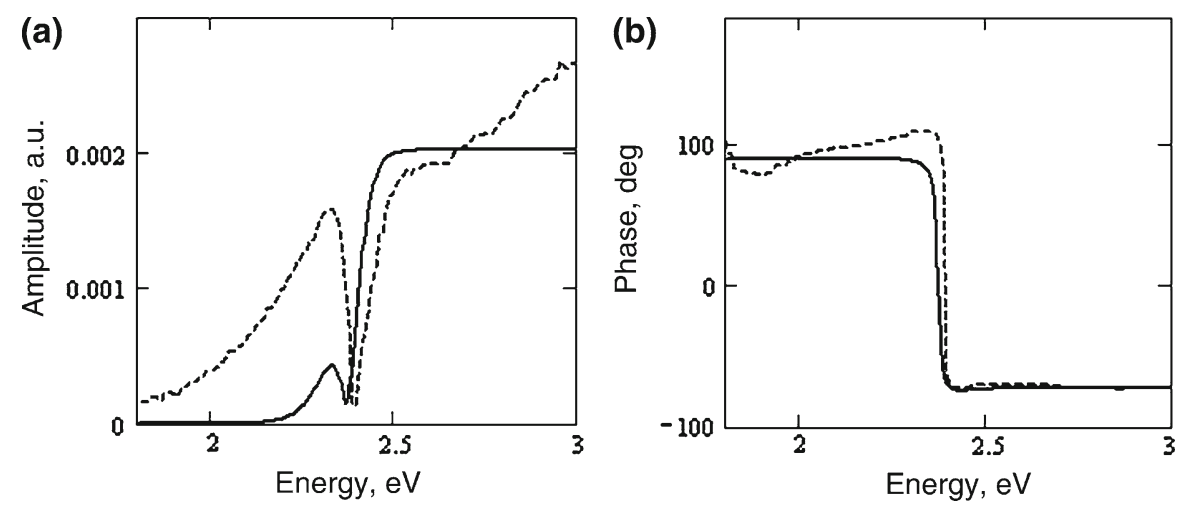

Fig. 1 Simulation of ideal sample spectra (solid line) and experimental data (dashed line) of CdS: (a) amplitude and (b) phase 

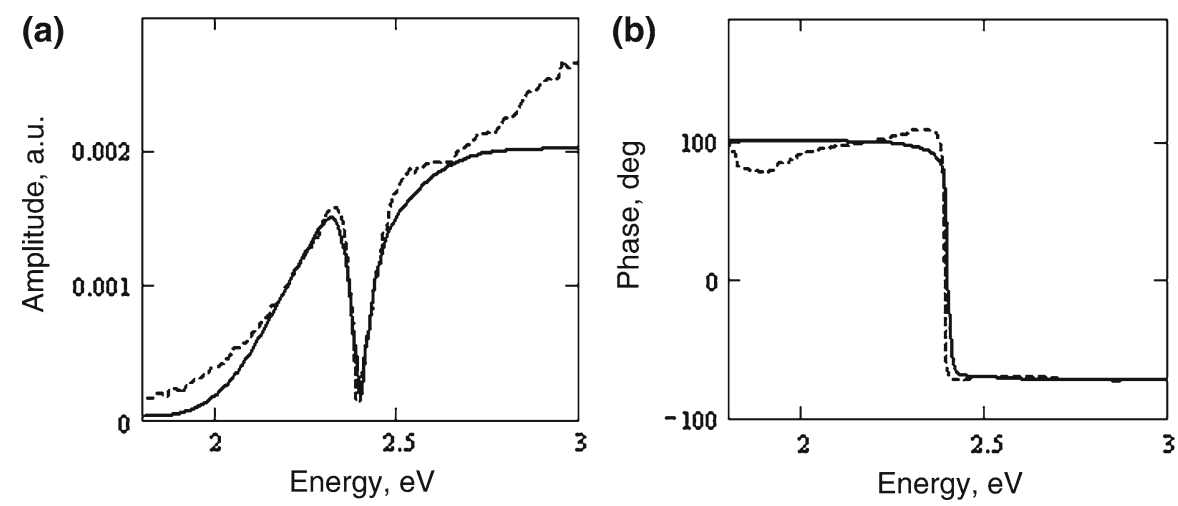

Fig. 2 Simulation of the spectra in the presence of surface defects (solid line) and experimental data (dashed line) of CdS: (a) amplitude and (b) phase
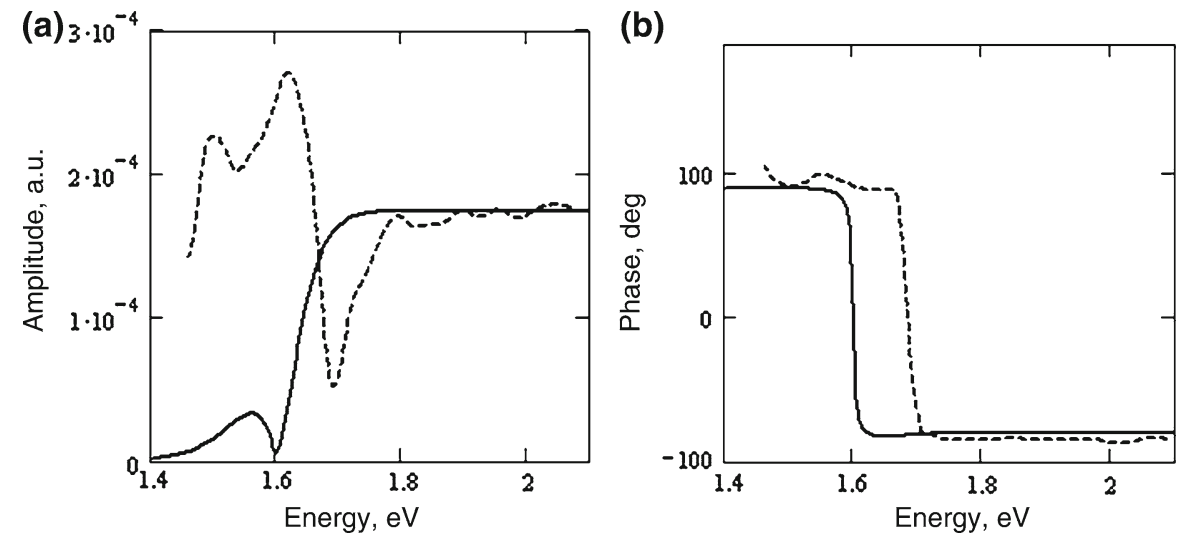

Fig. 3 Simulation of ideal sample spectra (solid line) and experimental data (dashed line) of CdSe: (a) amplitude and (b) phase

and phase spectra of simulation are moved to the lower energy in comparison to the experiment. For the value of the energy equal to the energy gap of CdSe where the amplitude bends and still increases, saturation occurs for an energy near $1.8 \mathrm{eV}$. In the sub-bandgap region, a maximum larger than the value of the amplitude at saturation is observed.

Figure $4 \mathrm{a}, \mathrm{b}$ shows the simulation and experimental results for the case if one assumes a layer of thickness $d=0.01 \mathrm{~mm}$ (the rest of the parameters are the same as before) and two defects of energies: $E_{\mathrm{d} 1}=1.62 \mathrm{eV}$ and $E_{\mathrm{d} 2}=1.68 \mathrm{eV}$, both located on the illuminated surface. Both the phase and amplitude move to the higher energy, and the characteristic bending at the amplitude is observed close to the energy bandgap value. The model used in the simulation contains two possible defects at the layer of the lower value of the thermal conductivity in comparison to the volume of the sample. Additional assumptions must be taken into account to simulate the maximum at an energy of $1.5 \mathrm{eV}$. 

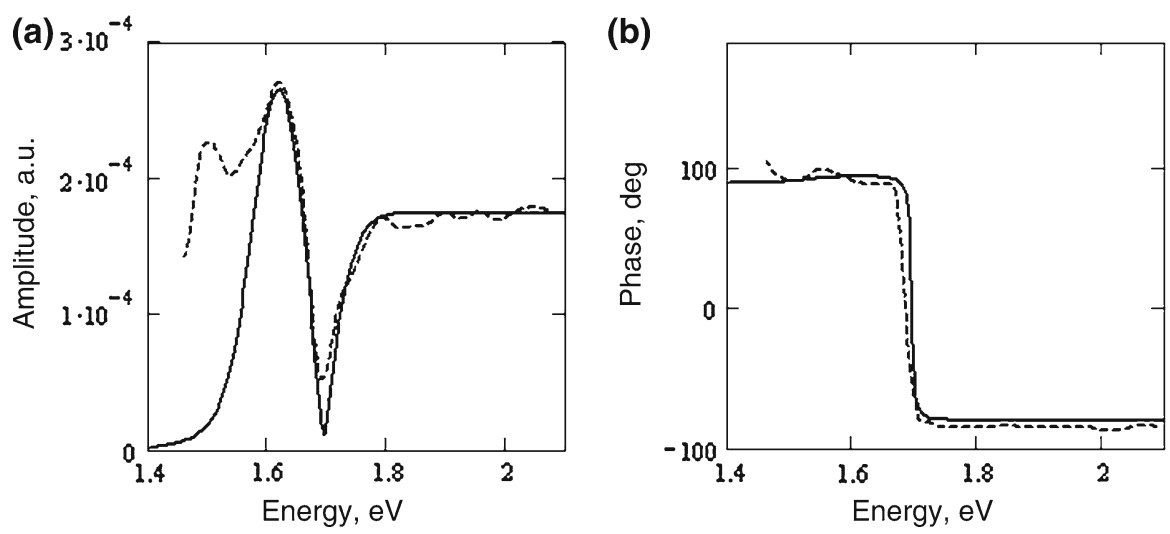

Fig. 4 Simulation of the spectra in the presence of surface defects (solid line) and experimental data (dashed line) of CdSe: (a) amplitude and (b) phase

\section{Conclusions}

The samples were prepared by polishing to obtain a surface of good quality. This simple procedure did not cause the surfaces to be ideal. Experimental results of the investigated materials do not show a character consistent with the theories of J-A or Blonskij. These theories may be used, however, with the additional assumption of the presence of a surface defect. The influence of the surface manifests in different ways for different materials and may be interpreted by the modified Blonskij model. This is a good basis to obtain information on the surface quality and investigation of surface preparation processes. The assumptions showed the influence of the defect levels on the amplitude and phase piezoelectric spectra. To determine the exact values of the thickness of the layer and changes in the thermal diffusivity of the layer, comparisons to other methods and multiparameter fitting procedures must be carried out.

Open Access This article is distributed under the terms of the Creative Commons Attribution License which permits any use, distribution, and reproduction in any medium, provided the original author(s) and the source are credited.

\section{References}

1. P. Wang, T. Nakagawa, A. Fukuyama, K. Maeda, Y. Iwasa, M. Ozeki, Y. Akashi, T. Ikari, Mater. Sci. Eng. C 26, 826 (2006)

2. A. Fukuyama, S. Sakamoto, S. Sonoda, P. Wang, K. Sakai, T. Ikari, Thin Solid Films 112, 511 (2006)

3. K. Sakai, T. Kakeno, T. Ikari, S. Shirakata, T. Sakemi, K. Awai, T. Yamamoto, J. Appl. Phys. 99, 043508 (2006)

4. K. Yoshino, H. Komaki, T. Kakeno, Y. Akaki, T. Ikari, J. Phys. Chem. Solids 64, 1839 (2003)

5. T. Hoshimiya, NDT \& E Int. 32, 133 (1999)

6. K.S. Katti, M.W. Urban, Polymer 44, 3319 (2003)

7. N. Takabatake, T. Kobayashi, Y. Show, T. Izumi, Mater. Sci. Eng. B 91-92, 186 (2002)

8. S. Poulin, D.Q. Yang, E. Sacher, C. Hyett, T.H. Ellis, Appl. Surf. Sci. 165, 15 (2000)

9. W. Jackson, N.M. Amer, J. Appl. Phys. 51, 3343 (1980)

10. I.V. Blonskij, V.A. Thoryk, M.L. Shendeleva, J. Appl. Phys. 79, 3512 (1996) 
11. M. Maliński, J. Zakrzewski, K. Strzałkowski, Int. J. Thermophys. 28, 299 (2007)

12. M. Maliński, J. Zakrzewski, K. Strzałkowski, S. Łęgowski, F. Firszt, H. Męczyńska, Surf. Sci. 603, 131 (2009)

13. J. Zakrzewski, M. Maliński, K. Strzałkowski, F. Firszt, S. Łęgowski, H. Męczyńska, Int. J. Thermophys. 31, 208 (2010)

14. M. Maliński, J. Zakrzewski, Eur. Phys. J. Spec. Top. 154, 345 (2008)

15. M. Maliński, L. Bychto, J. Zakrzewski, J. Phys. IV 129, 245 (2005) 